Schmerz 2021 · 35:391-400

https://doi.org/10.1007/s00482-021-00550-9

Eingegangen: 6 . November 2020

Überarbeitet: 17. Februar 2021

Angenommen: 19. Februar 2021

Online publiziert: 20. April 2021

(c) Der/die Autor(en) 2021

\author{
M. I. Emons ${ }^{1}$ T. H. Scheeper-von der Born ${ }^{1}$ F. Petzke' $\cdot$ V. Ellenrieder ${ }^{2}$. \\ L. Reinhardt ${ }^{3} \cdot$ W. Meißner ${ }^{4,5} \cdot$ J. Erlenwein ${ }^{1}$ \\ 'Klinik für Anästhesiologie, Universitätsmedizin Göttingen, Göttingen, Deutschland \\ ${ }^{2}$ Klinik für Gastroenterologie und gastrointestinale Onkologie, Universitätsmedizin Göttingen, Göttingen, \\ Deutschland \\ ${ }^{3}$ Klinik für Innere Medizin, Eichsfeld Klinikum, Heilbad Heiligenstadt, Deutschland \\ ${ }^{4}$ Klinik für Anästhesiologie und Intensivmedizin, Universitätsklinikum Jena, Jena, Deutschland \\ ${ }^{5}$ Klinik für Innere Medizin II, Abteilung Palliativmedizin, Universitätsklinikum Jena, Jena, Deutschland
}

\section{Schmerzmanagement in der Inneren Medizin}

\section{Ergebnisse einer bundesweiten Erhebung zu Struktur- und Prozessdaten}

\section{Einleitung}

Seit vielen Jahren werden durch nationale und internationale Erhebungen anhaltende Defizite in der Qualität der Schmerztherapie im Krankenhaus beschrieben [1, 16, 24, 25]. Diese Aussagen basieren jedoch meist auf Daten von operativen Patienten und nur wenige Untersuchungen beziehen sich auf die Versorgungssituation nichtoperativer Patienten. Die verfügbaren Erhebungen zeigen, dass Defizite in der Versorgungsqualität im konservativen Bereich denen der operativen Bereiche entsprechen [14, 20, 24]. Die Analyse von Teilaspekten lässt sogar darauf schließen, dass die Unter- und Fehlversorgung mit ausbleibender oder verzögerter Therapie von Schmerzen in den nichtoperativen Bereichen noch ausgeprägter ist [24]. Ein Bericht (Health Technology Assessment [HTA]) im Auftrag des Deutschen Instituts für Medizinische Dokumentation und Information und des Bundesgesundheitsministeriums bestätigt diese Einschätzung [20]. Versorgungsdefizite des Schmerzmanagements basieren mit auf fehlenden Regelungen zu Behandlungsabläufen und Verantwortlichkeit [10, 20, 26].

Erhebungen zur Akutschmerztherapie aus Sicht von Chirurgen oder Anästhesisten wurden bereits mehrfach durchgeführt [10, 21, 27, 30]. Informa- tionen zu Strukturen und Prozessen des Schmerzmanagements in nichtoperativen Fachabteilungen lagen bisher nur aus zweiter Hand durch die Befragung anästhesiologischer Chefärzte vor und basierten nicht auf Angaben der Internisten selbst [13]. Bereits im Jahr 2016 sollten, analog zu den Vorbefragungen für die operative Medizin, in einem gemeinsamen Projekt zwischen Deutscher Gesellschaft für Innere Medizin e.V. (DGIM), der Deutschen Schmerzgesellschaft e. V. und unserer Arbeitsgruppe Struktur- und Prozessdaten erfasst werden. Hierzu wurden im Jahr 2016 alle als Chefärzte/leitende Ärzte registrierten Mitglieder der DGIM von ihrer Gesellschaft mit der Bitte um Teilnahme angeschrieben, inkl. einmaligen Reminders. Aufgrund des Rücklaufs von insgesamt 34 Fragebögen wurden Auswertung und Veröffentlichung verworfen.

Ziel des vorliegenden Projekts unserer Arbeitsgruppe war es, erneut aktuelle Struktur- und Prozessdaten des Schmerzmanagements in internistischen Fachabteilungen deutscher Krankenhäuser zu gewinnen. Aufgrund der Vorerfahrungen erfolgte die Datenerfassung jedoch mit adaptierter Erhebungsmethodik. Nichtuniversitäre Abteilungen wurden nach persönlicher Kontaktaufnahme per Telefoninterview befragt - aufgrund der großen Anzahl von Abteilungen allerdings anhand einer randomisiert ausgewählten Stichprobe. Da aus unserer Sicht aufgrund des meist höheren Spezialisierungsgrads und der damit verbundenen Heterogenität eine randomisierte Auswahl der universitären internistischen Abteilungen nicht sinnvoll erschien, wurden alle Einrichtungen persönlich angeschrieben und erhielten einen Link für eine Onlineerfassung der Fragen.

\section{Methodik und Materialien}

\section{Kohorte und Randomisierung}

Die Datenerhebung der Struktur- und Prozessdaten der nichtuniversitären Fachabteilungen für innere Medizin erfolgte prospektiv und randomisiert zwischen Juni und Oktober des Jahres 2018. Anhand des „Deutschen Krankenhausverzeichnisses“ (https://www. deutsches-krankenhaus-verzeichnis.de/, Stand 05.07.2017) wurden jeweils bundeslandspezifisch insgesamt 1317 nichtuniversitäre Krankenhäuser mit mindestens einer internistischen Fachabteilung ermittelt [4]. Ziel war es, für eine repräsentative Darstellung der Versorgungssituation hiervon ca. ein Drittel einzuschließen (450 Krankenhäuser). Die Auswahl der einzuschließenden Krankenhäuser erfolgte per Randomisierungsfunktion des Programms Excel (Microsoft Corporation, Redmond, 
USA, Version 2016) und konsekutiver Auswahl der in der entstehenden Reihung ersten 450 Kliniken. Anschließend wurden Kontaktdaten aller internistischen Einzelabteilungen der ausgewählten Krankenhäuser anhand einer Onlinerecherche erfasst (726 Abteilungen an 450 Krankenhäusern). 40 Abteilungen mussten ausgeschlossen werden, da die Angaben des Krankenhausverzeichnisses nicht mehr aktuell waren und zwischenzeitlich Schließungen oder Fusionierungen erfolgt waren, es sich um angeschlossene Praxen handelte oder um Krankenhäuser, für die in der Onlinerecherche keine zugehörige internistische Abteilung ermittelt werden konnte.

Nach Randomisierung und Onlinerecherche wurden für die einzuschließenden nichtuniversitären Krankenhäuser 686 eigenständige internistische $\mathrm{Ab}$ teilungen ermittelt, und es erfolgte $\mathrm{zu}$ nächst eine schriftliche Ankündigung der Studie sowie nachfolgend die telefonische Kontaktaufnahme.

\section{Datenerfassung}

Die Kontaktaufnahme und Befragung der internistischen Abteilungen erfolgte von Juni 2018 bis Oktober 2018. Zur Verbesserung des Rücklaufs erfolgte die Kontaktaufnahme adaptiert an die jeweiligen Bundesländer unter Umgehung der Ferienzeiten. Per E-Mail wurden die Chefärzte der Abteilungen jeweils ca. 2 Wochen vor Kontaktaufnahme angeschrieben und die Befragung angekündigt. Die telefonische Kontaktaufnahme erfolgte dann entsprechend im Abstand dieses Zeitfensters an Werktagen zu Geschäftszeiten, vorzugsweise vormittags. Abteilungen, die $\mathrm{zu}$ drei unterschiedlichen Zeitpunkten nicht telefonisch erreichbar waren, wurden nicht weiter kontaktiert.

\section{Erfassungsparameter}

Für eine bessere Vergleichbarkeit mit bestehenden Daten für die operative Versorgung erfolgte die Erfassung mit einer reduzierten und in einigen Punkten angepassten Version des Akutschmerzzensus (2012), basierend auf den organisatorischen Eckpunkten der S3-Leitlinie
„Behandlung akuter perioperativer und posttraumatischer Schmerzen“ ([22]; z.B. Regelungen von Verantwortlichkeiten, Rahmenvereinbarungen, Implementierung von Akutschmerzdiensten, -erfassung und -dokumentation, Konzepte zur Prophylaxe und Therapie). Die Datenerfassung erfolgte per standardisiertem Telefoninterview. Es wurden folgende Parameter erfasst:

1. Allgemeine Angaben zum Krankenhaus (Versorgungsstufe, Trägerschaft)

2. Angaben zur Abteilung für innere Medizin (Subspezialisierung, Bettenzahl, Position des Antwortenden)

3. Schmerzerfassung und Dokumentation (Frequenz der Schmerzerfassung, Effektivitätskontrolle nach Bedarfsanalgesie, Verfahren zur Schmerzerfassung, Art und Weise der Dokumentation)

4. Behandlungsstandards (Bedarfsmedikation, Handlungs- bzw. Interventionstrigger zur Bedarfsanalgesie, Verantwortlichkeit für die regelmäßige Überarbeitung der Standards, Einbindung nichtmedikamentöser Verfahren)

5. Rahmenbedingungen des Schmerzmanagements und spezielle Versorgungsstrukturen (Vorhandensein schriftlicher Vereinbarungen zur Zusammenarbeit in der Schmerztherapie, Einbindung von Schmerzdienst/ schmerztherapeutischer Beratung)

6. Qualitätssicherung und Zertifizierung (regelmäßige Auswertung von Ergebnisdaten zur Darstellung der Ergebnisqualität, hausinterne Qualitätszirkel, Zertifizierung des Schmerzmanagements [Certkom, TÜV Rheinland], Implementierung des Expertenstandards „Schmerzmanagement in der Pflege“).

\section{Struktur- und Prozessdaten universitärer internistischer Fachabteilungen}

In einem zweiten Schritt erfolgte die Datenerfassung bei universitären internistischen Fachabteilungen. Diese erfolgte per Onlinebefragung anhand desselben Fragenkatalogs. Die Erfassung der universitären Abteilungen erfolgte anhand
Onlinerecherche bei allen internistischen Abteilungen der 34 Vollmitglieder des Verbands der Universitätsklinika Deutschlands e.V. (VUD) sowie drei Oldenburger Kliniken als assoziierte Mitglieder. Hierbei zeigte sich, dass ein universitäres Krankenhaus mit zwei Abteilungen sowie eine spezialisierte Fachabteilung bereits bei der Auflistung nichtuniversitärer Krankenhäuser selektiert wurden und diese bereits telefonisch befragt worden waren. Diese drei Abteilungen wurden nicht erneut kontaktiert und verblieben in der Kohorte der nichtuniversitären Abteilungen, da es sich nicht um Abteilungen eines langjährigen Universitätsklinikums handelte, sondern um Abteilungen universitärer Teilkrankenhäuser. Zwischen Januar und März 2019 wurden 195 universitäre internistische Abteilungen mittels persönlichen Anschreibens per Mail an die Direktoren kontaktiert und gebeten, die Onlinebefragung zu unterstützen. Es wurden zwei Reminder im Abstand von jeweils 3 Wochen versandt. Die Onlinebefragung erfolgte mittels der Onlinesoftware SurveyMonkey (SurveyMonkey, Inc, Palo Alto, Kalifornien, USA, 2019).

\section{Datenauswertung}

Nach der Datenerfassung erfolgte eine anonymisierte Analyse in beiden Studienarmen. Die Darstellung der Ergebnisse erfolgte deskriptiv und getrennt zwischen nichtuniversitären und universitären Abteilungen. Ziel der Befragung war die Darstellung des Schmerzmanagements sowohl in nichtuniversitären als auch in universitären Abteilungen. Ein Vergleich zwischen nichtuniversitären und universitären Abteilungen war nicht beabsichtigt. Die Darstellung erfolgt deshalb getrennt. Prozentangaben wurden gerundet. Sofern nicht anders angegeben, beziehen sich die Angaben auf die eingeschlossenen Abteilungen. Die Auswertung der Daten erfolgte mit SPSS (IBM, Armonk, USA, Version 25). 
Schmerz 2021 · 35:391-400 https://doi.org/10.1007/s00482-021-00550-9

(c) Der/die Autor(en) 2021

M. I. Emons · T. H. Scheeper-von der Born · F. Petzke · V. Ellenrieder · L. Reinhardt · W. Meißner · J. Erlenwein

\section{Schmerzmanagement in der Inneren Medizin. Ergebnisse einer bundesweiten Erhebung zu Struktur- und Prozessdaten}

\section{Zusammenfassung}

Hintergrund und Ziel der Arbeit. Seit Jahren werden Defizite der Qualität der Schmerztherapie im Krankenhaus beschrieben. Ziel der vorliegenden Untersuchung war es, Strukturen und Prozesse des Schmerzmanagements in internistischen Abteilungen darzustellen. Material und Methoden. Die Datenerfassung erfolgte mittels eines standardisierten Telefoninterviews (nichtuniversitäre Abteilungen); bei universitären Abteilungen separat mittels eines Onlinefragebogens (SurveyMonkey ${ }^{\circledR}$ ). Ergebnisse. Daten von 139 nichtuniversitären Abteilungen (Rücklauf: $21 \%$ ) und 33 universitären Abteilungen (davon 21 vollständig beantwortete Fragebögen, Rücklauf $17 \%$ bzw. $11 \%$ ) wurden erhoben. 441 von 619 kontaktierten nichtuniversitären Abteilungen lehnten die Teilnahme ausdrücklich ab, am häufigsten mit der Begründung, es bestünde kein Interesse am Thema Schmerzmanagement. In den 172 teilnehmenden Einrichtungen wurde Schmerz als eigenständiger Parameter in $89 \%$ der nichtuniversitären Abteilungen (96\% universitär) regelmäßig während der Visite erfasst; schriftliche Behandlungsstandards zur Schmerztherapie lagen in 57\% der nichtuniversitären Abteilungen vor (54\% universitär). In $76 \%$ der nichtuniversitären Krankenhäuser (100\% universitär) stand auch für die internistischen Patienten ein Akutschmerzdienst zur Mitbehandlung zur Verfügung, schriftliche Vereinbarungen zur Zusammenarbeit lagen bei $35 \%$ vor (18\% universitär).
Diskussion. Die berichtete Umsetzung des Schmerzmanagements war in den teilnehmenden Abteilungen gut. Gleichwohl sind die Ergebnisse bei niedriger Teilnahme und häufiger Ablehnung mit der Begründung einer fehlenden Relevanz nur eingeschränkt aussagekräftig. Die erfassten Daten reflektieren daher vermutlich eine erhebliche positive Selektion und sind nicht als repräsentativ für das Schmerzmanagement in der inneren Medizin zu werten.

\section{Schlüsselwörter}

Akutschmerzmanagement · Akutschmerzdienst - Behandlungsstandards - Schmerzmedizin · Innere Medizin

\section{Pain management in departments of internal medicine. Results of a national survey on structures and processes of care}

\section{Abstract}

Background, Objectives. Deficits in the quality of pain management in hospitals have been described for years. The aim of this study was to assess structures and processes of pain management in departments for internal medicine in German hospitals.

Materials and methods. Data were collected using a standardized telephone interview (non-university hospital departments) on a randomized sample of hospitals (circa 1/3 of hospitals); all German departments of university hospitals were invited to participate and questioned separately using an online questionnaire (SurveyMonkey ${ }^{\circledR}$ ).

Results. Data from 139 non-university departments (response rate: $21 \%$ ) and 33 university hospital departments (only 21 questionnaires were fully answered, response rate 17 and $11 \%$, respectively) were collected. Of 619 non-university hospital departments contacted, 441 explicitly refused to participate in the survey, most often on the grounds that there was no interest in pain management. Pain was regularly recorded as an independent parameter during the medical visit in $89 \%$ of the non-university hospital and $96 \%$ of the university hospital departments; written standardized treatment protocols for pain therapy were available in $57 \%$ of the non-university hospital departments (54\% university hospital departments). In $76 \%$ of the non-university hospitals departments (100\% university hospital departments), an acute pain service was also available for patients of internal medicine departments for co-treatment. Written cooperation agreements were less common $35 \%$ nonuniversity hospital departments, $18 \%$ university hospital departments). Conclusion. The described implementation of pain management was satisfying in the participating departments. However, in consideration of the low participation and high rejection of participation due to explicit disinterest in the topic, the results should be critically assessed and presumably characterized by a significant positive bias.

\section{Keywords}

Acute pain management - Acute pain service . Standards of care $\cdot$ Pain medicine $\cdot$ Internal medicine

\section{Ergebnisse}

\section{Einschlüsse der nichtuniversitären Krankenhäuser}

Von den 686 eigenständigen internistischen Abteilungen nahmen 139 an der Befragung teil (• Abb. 1). Abteilungen, welche die Teilnahme aktiv ablehnten, begründeten dies am häufigsten ausdrück- lich mit fehlendem Interesse am Thema Schmerzmanagement $(n=312)$, gefolgt von Zeit- $(n=119)$ und Personalmangel $(n=43) .8$ Abteilungen gaben an, generell nicht an Befragungen teilzunehmen. Drei Abteilungen gaben als Begründung für die Nichtteilnahme an, keine Schmerzbehandlung zu betreiben (Mehrfachantworten möglich).
Die Interviewpartner waren in den meisten Fällen Oberärzte $(40 \%, n=56)$ oder Chefärzte $(32 \%, n=45)$, gefolgt von Weiterbildungsassistenten $(20 \%, n=28)$ und Fachärzten $(6 \%, n=8)$, sowie in je einem Fall eine Dokumentationsassistentin und eine Stationspsychologin (je 1\%). Die Strukturcharakteristika der teilnehmenden Abteilungen stellt $\bullet$ Tab. 1 dar. 


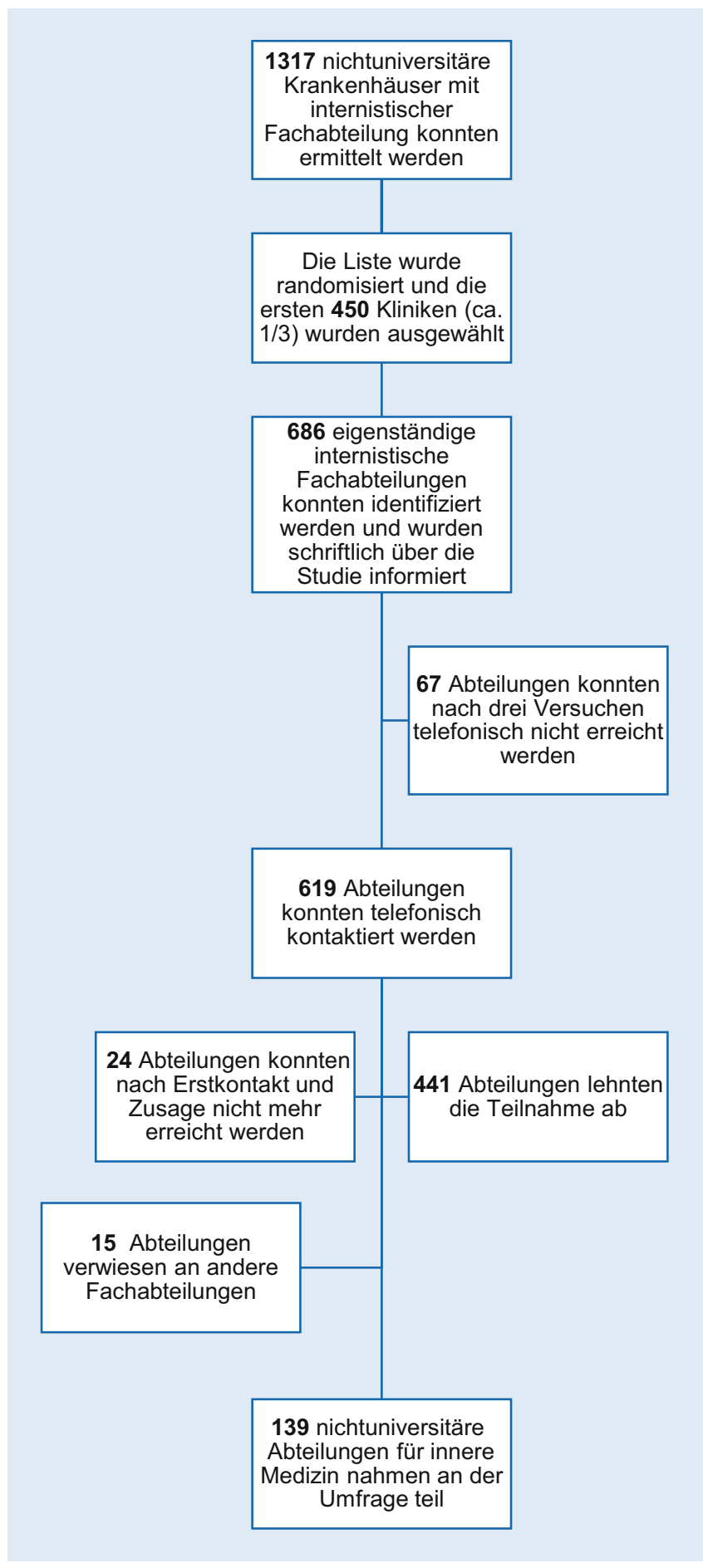

Abb. $1 \triangleleft$ Einschlüsse der nichtuniversitären Abteilungen
Die Spezialisierungen der teilnehmenden Abteilungen sind in $\bullet$ Tab. 2 zusammengefasst.

\section{Schmerzerfassung und Dokumentation (nichtuniversitäre Krankenhäuser)}

Von den befragten nichtuniversitären Abteilungen gaben $89 \%$ an, dass „Schmerz“ als eigenständiger Parameter während der ärztlichen Visite und/oder der Pflegevisite regelhaft erfasst wird $(n=124)$. In den meisten Abteilungen erfolgt dies laut Angaben mindestens einmal am Tag (38\%, $n=53$; mind. $2 \times$ täglich: $17 \%, n=24 ;$ „3 $\times$ täglich oder mindestens einmal pro Schicht“: $19 \%$, $n=26$; „ab und zu“: $8 \%, n=11)$. In $76 \%$ der Abteilungen $(n=105)$ wurde außerhalb der Routineerfassung (z.B. bei Patientenmeldung wegen Schmerz) die Schmerzintensität „meistens oder immer" erhoben ( $n=105$; , ab und zu“: $19 \%, n=26$; keine Schmerzmessung außerhalb der Routineerfassung: 6\%, $n=8)$. Zur Kontrolle nach einer medikamentösen Schmerztherapie wurde die Schmerzintensität nach Angaben der Befragten in etwa der Hälfte der Abteilungen „meistens oder immer" erneut gemessen ( $50 \%, n=70$; „ab und $\mathrm{zu}$ “: $25 \%, n=35$; „nie“: $25 \%, n=34)$. Zur Schmerzerfassung wurde in der Mehrheit der Abteilungen ein standardisiertes Verfahren genutzt $(90 \%, n=125)$.

Zur Effektivitätskontrolle der Schmerztherapie (Dokumentation durch die Stationspflege mind. $1 \times$ täglich, Mehrfachantworten möglich) wurde am häufigsten der Analgetikaverbrauch dokumentiert und evaluiert $(86 \%, n=120)$, gefolgt von der Schmerzintensität in Ruhe $(81 \%, n=113)$ und von Nebenwirkungen (77\%, $n=107)$. Die Vigilanz bzw. der Sedierungsgrad wurde in $44 \%$ der Abteilungen $(n=61)$ mindestens $1 \times$ täglich dokumentiert.

\section{Behandlungsstandards und Verantwortlichkeiten (nichtuniversitäre Krankenhäuser)}

Schriftliche Standards zur Behandlung von Schmerzen lagen zum Befragungszeitpunkt in $57 \% \quad(n=79)$ vor (nur mündliche Vereinbarungen: $23 \%, n=32$; keine Standards/Konzepte: $20 \%, n=28$ ) und beinhalteten, wenn vorhanden, bis auf wenige Ausnahmen die Gabe einer Bedarfsmedikation (95\%, $n=75$ ). Allerdings enthielten diese Standards nur in etwa der Hälfte der Fälle auch einen Interventionstrigger für die Gabe einer Bedarfsmedikation $(53 \%, n=42)$. Nichtmedikamentöse Therapieverfahren waren in $47 \%$ der Abteilungen mit schriftlichen Standards in diesen enthalten $(n=37)$. 
Tab. 1 Strukturdaten der nichtuniversitären Krankenhäuser

\begin{tabular}{ll|l|l}
\hline Versorgungsstufe & & Trägerschaft \\
\hline Grundversorgung & $19 \%(n=27)$ & Öffentlich & $35 \%(n=48)$ \\
\hline Regel-/Schwerpunktversorgung & $52 \%(n=72)$ & Freigemeinnützig & $39 \%(n=54)$ \\
\hline Maximalversorgung & $19 \%(n=27)$ & Privat & $27 \%(n=37)$ \\
\hline Fach- und/oder Belegklinik & $8 \%(n=11)$ & - & - \\
\hline Sonstiges & $1 \%(n=2)$ & - & -
\end{tabular}

Tab. 2 Spezialisierung der teilnehmenden Abteilungen

Spezialisierung der Abteilung Nichtuniversitäre Abteilungen ${ }^{a}$ Universitäre Abteilungen

\begin{tabular}{|l|l|l}
\hline Allgemeine innere Medizin & $35 \%(n=48)$ & - \\
\hline Gastroenterologie & $27 \%(n=38)$ & $18 \%(n=6)$ \\
\hline Kardiologie & $22 \%(n=30)$ & $4 \%(n=1)$ \\
\hline Geriatrie & $23 \%(n=32)$ & - \\
\hline Hämatologie-Onkologie & $14 \%(n=20)$ & $29 \%(n=8)$ \\
\hline Pneumologie & $8 \%(n=11)$ & $11 \%(n=3)$ \\
\hline Rheumatologie & $4 \%(n=6)$ & $11 \%(n=3)$ \\
\hline $\begin{array}{l}\text { Nephrologie } \\
\text { Endokrinologie }\end{array}$ & $4 \%(n=6)$ & $18 \%(n=5)$ \\
\hline $\begin{array}{l}\text { Angiologie } \\
\text { Sonstiges }\end{array}$ & $3 \%(n=4)$ & $7 \%(n=2)$ \\
\hline $\begin{array}{l}\text { a Sonstiges: } n=4 \text { : Palliativmedizin, } \\
\text { Infektiologie }\end{array}$ & - & $21 \%\left(n=7^{\text {a }}\right)$ \\
\hline
\end{tabular}

Die Befragten gaben an, dass in $25 \%$ der Abteilungen $(n=34)$ ausschließlich der Stationsarzt für das Schmerzmanagement verantwortlich war. In $40 \%$ der Abteilungen $(n=56)$ stand zudem ein Akutschmerzdienst oder Schmerzdienst konsiliarisch zur Verfügung. In $35 \%(n=49)$ der Abteilungen gab es die Möglichkeit der aktiven Mitbehandlung durch einen Akutschmerzdienst, zum Beispiel durch Betreuung invasiver Verfahren. Zur Beratung bei komplexen schmerzmedizinischen bzw. palliativmedizinischen Fragestellungen stand in der überwiegenden Mehrzahl der Kliniken ein schmerzmedizinischer ( $87 \%, n=121)$ oder palliativmedizinischer Konsildienst $(48 \%, n=67)$ zur Verfügung. Schriftliche Vereinbarungen zur Zusammenarbeit im Schmerzmanagement zwischen der schmerzmedizinischen Abteilung/dem Schmerzdienst und den internistischen Fachabteilungen lagen zum Zeitpunkt der Befragung in einem Drittel $(35 \%, n=49)$ der Abteilungen vor.
Qualitätsmanagement im Kontext Schmerztherapie (nichtuniversitäre Krankenhäuser)

Der Anteil der Abteilungen, in denen das Schmerzmanagement laut der Befragten zertifiziert war, lag bei $8 \%$. Gut die Hälfte gab an, dass ein solches Zertifikat geplant sei. In einem knappen Drittel war der Expertenstandard Schmerzmanagement in der Pflege implementiert. In $40 \%$ der Kliniken gab es einen Qualitätszirkel, der sich mit der Akutschmerztherapie befasst und an dem sich in gut $2 / 3$ der Fälle auch internistische Kollegen beteiligten (- Tab. 3).

\section{Schmerzmanagement in internistischen Abteilungen der universitären Medizin}

Von den universitären Abteilungen nahmen 33 von 195 angeschriebenen Abteilungen $(17 \%)$ an der Onlinebefragung teil. Die vollständige Beantwortung der Fragen fiel im Verlauf des Fragebogens ab. Nur 21 der Abteilungen schlossen die Befragung auch ab (Rücklauf für komplettierte Fragebögen:
$11 \%$ der kontaktierten Abteilungen). Aufgrund der anonymen Onlinebefragung erfolgte keine Erfassung für Gründe einer ausbleibenden oder abgebrochenen Teilnahme. Die meisten universitären Abteilungen gehörten einem Klinikum mit über 1000 Betten an (73\%, $n=24$; 700-999 Betten: $24 \%, n=8$; 400-699 Betten: $3 \%, n=1)$. Beantwortet wurde der Fragebogen in den meisten Fällen von einem Oberarzt ( $44 \%$, $n=14)$, seltener vom Direktor/Chefarzt/ Abteilungsleiter (19\%, $n=6)$, gefolgt von Assistenten in der Weiterbildung (16\%, $n=5)$ und Fachärzten $(9 \%, n=3)$. In $12 \%$ $(n=4)$ der Fälle wurde der Fragebogen von nichtärztlichen Kollegen beantwortet (jeweils $1 \times$ genannt: „Bereichsleitung“, „Study Nurse“, „Gesundheits-/ Krankenpflegerin“, „Studienkoordinatorin“, fehlend: $n=1$ ). Die Ergebnisse für die universitären Abteilungen sind in - Tab. 2, 3, 4, 5 und 6 dargestellt.

Insgesamt wurde Schmerz als eigenständiger Parameter etwas häufiger erfasst als in den nichtuniversitären Abteilungen. Außerdem standen in den universitären Abteilungen in allen Fällen ein Akutschmerzdienst sowie ein palliativmedizinischer Konsildienst zur Verfügung. Auch bei den Angaben universitärer Häuser zum Qualitätsmanagement war der Anteil der Kliniken, die nach Einschätzung der Befragten Qualitätssicherung der Schmerztherapie betrieben, das Schmerzmanagement zertifiziert hatten bzw. ein Zertifikat anstrebten, wie bereits bei den nichtuniversitären Krankenhäusern hoch (• Tab. 3).

\section{Diskussion}

\section{Repräsentativität und Limitationen}

Zur Erfassung von Strukturen und Prozessen des Schmerzmanagements in internistischen Abteilungen wurden alle internistischen Abteilungen einer randomisierten Stichprobe von ca. 1/3 der nichtuniversitären Kliniken in Deutschland sowie alle universitären internistischen Abteilungen kontaktiert und zur Teilnahme an der Erfassung aufgefordert. Die Ergebnisse der nichtuniversitären Kliniken stellen einen bundesweiten Querschnitt hinsichtlich ihrer Vertei- 
Tab. 3 Zertifizierung und Qualitätssicherung des Schmerzmanagements

\begin{tabular}{|c|c|c|c|c|c|c|}
\hline \multirow[b]{2}{*}{-} & \multicolumn{3}{|c|}{ Nichtuniversitäre Abteilungen } & \multicolumn{3}{|c|}{ Universitäre Abteilungen } \\
\hline & $\mathrm{Ja}$ & Nein & Geplant & Ja & Nein & Geplant \\
\hline Schmerzmanagement des Hauses zertifiziert & $\begin{array}{l}8 \%^{\mathrm{a}} \\
(n=11)\end{array}$ & $\begin{array}{l}86 \%^{\mathrm{a}} \\
(n=119)\end{array}$ & $\begin{array}{l}7 \% \\
(n=9)\end{array}$ & $\begin{array}{l}50 \% \%^{b, c} \\
(n=11)\end{array}$ & $\begin{array}{l}32 \%^{\mathrm{b}} \\
(n=7)\end{array}$ & $\begin{array}{l}18 \% \\
(n=4)\end{array}$ \\
\hline $\begin{array}{l}\text { Abteilung ist innerhalb dieses Zertifikats mit zertifiziert (oder } \\
\text { dies wird angestrebt) }\end{array}$ & $\begin{array}{l}55 \%^{\mathrm{a}} \\
(n=6)\end{array}$ & $\begin{array}{l}45 \%^{\mathrm{a}} \\
(n=5)\end{array}$ & - & $\begin{array}{l}90 \% \text { b,d } \\
(n=9)\end{array}$ & $\begin{array}{l}10 \%{ }^{\mathrm{b}} \\
(n=1)\end{array}$ & - \\
\hline- & $\mathrm{Ja}$ & Nein & $\begin{array}{l}\text { Nicht be- } \\
\text { kannt }\end{array}$ & Ja & Nein & $\begin{array}{l}\text { Nicht be- } \\
\text { kannt }\end{array}$ \\
\hline $\begin{array}{l}\text { Daten zur Schmerztherapie werden mind. } 1 \text { ×/Jahr zur Qualitäts- } \\
\text { sicherung ausgewertet }\end{array}$ & $\begin{array}{l}25 \% \\
(n=34)\end{array}$ & $\begin{array}{l}75 \% \\
(n=105)\end{array}$ & - & $\begin{array}{l}32 \% \mathrm{e}^{2} \\
(n=6)\end{array}$ & $\begin{array}{l}68 \% \\
(n=13)\end{array}$ & - \\
\hline $\begin{array}{l}\text { Expertenstandard "Schmerzmanagement in der Pflege“ in der } \\
\text { Abteilung umgesetzt? }\end{array}$ & $\begin{array}{l}30 \% \\
(n=42)\end{array}$ & $\begin{array}{l}57 \% \\
(n=79)\end{array}$ & $\begin{array}{l}13 \% \\
(n=18)\end{array}$ & $\begin{array}{l}52 \%{ }^{f} \\
(n=11)\end{array}$ & $\begin{array}{l}14 \% \\
(n=3)\end{array}$ & $\begin{array}{l}33 \% \\
(n=7)\end{array}$ \\
\hline $\begin{array}{l}\text { Regelmäßig tagender interner Qualitätszirkel, der sich auch mit } \\
\text { der Akutschmerztherapie im Hause beschäftigt }\end{array}$ & $\begin{array}{l}40 \% \\
(n=55)\end{array}$ & $\begin{array}{l}36 \% \\
(n=50)\end{array}$ & $\begin{array}{l}25 \% \\
(n=34)\end{array}$ & $\begin{array}{l}24 \%^{f} \\
(n=5)\end{array}$ & $\begin{array}{l}24 \% \\
(n=5)\end{array}$ & $\begin{array}{l}52 \% \\
(n=11)\end{array}$ \\
\hline $\begin{array}{l}\text { Kollegen der Abteilung innere Medizin sind an diesem Zirkel } \\
\text { regelmäßig beteiligt }\end{array}$ & $\begin{array}{l}67 \% \\
(n=37)\end{array}$ & $\begin{array}{l}33 \% \\
(n=18)\end{array}$ & - & $\begin{array}{l}40 \% \\
(n=2)\end{array}$ & $\begin{array}{l}60 \% \\
(n=3)\end{array}$ & - \\
\hline 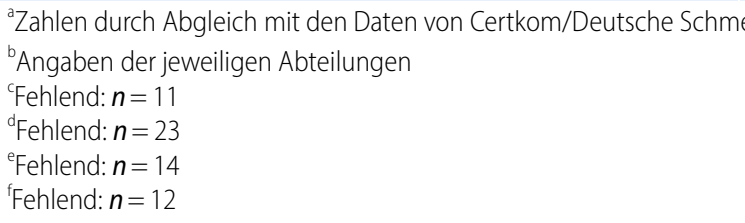 & erzgesellsc & te. V. und Tu & heinland er & & & \\
\hline
\end{tabular}

Tab. 4 Schmerzerfassung in den universitären Abteilungen

Erfassung von "Schmerz" während der Visite ${ }^{a}$

\begin{tabular}{|c|c|}
\hline $\mathrm{Ja}$ & $96 \%(n=26)$ \\
\hline Nein & $4 \%(n=1)$ \\
\hline \multicolumn{2}{|c|}{ Häufigkeit der Erfassung der Schmerzintensität ${ }^{b}$} \\
\hline $1 \times / \operatorname{Tag}$ & $39 \%(n=10)$ \\
\hline $3 \times / \operatorname{Tag}$ & $31 \%(n=8)$ \\
\hline $2 \times /$ Tag & $12 \%(n=3)$ \\
\hline „Ab und zu“ & $12 \%(n=3)$ \\
\hline Sonstiges & $8 \%(n=2)$ \\
\hline
\end{tabular}

Erfassung der Schmerzintensität durch ein standardisiertes Verfahren ${ }^{b}$

\begin{tabular}{|c|c|}
\hline Ja & $89 \%(n=23)$ \\
\hline Nein & $12 \%(n=3)$ \\
\hline \multicolumn{2}{|l|}{ Dokumentation der Schmerzerfassung ${ }^{b}$} \\
\hline In der Patientenkurve & $73 \%(n=19)$ \\
\hline Spezifische Akutschmerzdokumentation & $4 \%(n=1)$ \\
\hline Keine syst. Dokumentation & $19 \%(n=5)$ \\
\hline Sonstiges & $4 \%(n=1)$ \\
\hline \multicolumn{2}{|c|}{ Erhebung der Schmerzintensität außerhalb Routineerfassung (z. B. Patientenmeldung) } \\
\hline Meistens/immer & $69 \%(n=18)$ \\
\hline Ab und zu & $27 \%(n=7)$ \\
\hline Nie & $4 \%(n=1)$ \\
\hline \multicolumn{2}{|c|}{ Erfassung der Schmerzintensität nach Verabreichen von Bedarfsmedikation ${ }^{b}$} \\
\hline Meistens/immer & $58 \%(n=15)$ \\
\hline $\mathrm{Ab}$ und zu & $38 \%(n=10)$ \\
\hline Nie & $4 \%(n=1)$ \\
\hline $\begin{array}{l}{ }^{\mathrm{a}} \text { Fehlend: } n=6 \\
{ }^{\mathrm{b}} \text { Fehlend: } n=7\end{array}$ & \\
\hline
\end{tabular}

lung auf die einzelnen Bundesländer, die Krankenhausträger und Häuser unterschiedlicher Bettenanzahl dar [5]. Dennoch sind die Aussagen - wie es typisch für derartige Befragungserhebungen ist in ihrer Repräsentativität limitiert. Dabei ist insbesondere ein erheblicher positiver Selektionsbias zu vermuten. Dies zeigt sich in einer großen Diskrepanz zwischen hohem Anteil an Ablehnungen bzw. ausdrücklichem Desinteresse am Thema Schmerzmanagement auf der einen und hoher Qualität der Strukturund Prozessdaten der teilnehmenden Abteilungen auf der anderen Seite. Dies lässt vermuten, dass die Antworten zu einem großen Teil aus stärker engagierten Einrichtungen und von Kollegen, die für das Thema Schmerzmanagement sensibilisiert sind, stammten.

Nachdenklich macht nicht nur die Erfahrung aus der ersten Onlinebefragung (siehe oben), sondern auch der hohe Anteil an Abteilungen, die nicht nur - wie bei Befragungen mit teils vergleichbarer Rücklaufquote durchaus üblich - nicht teilnahmen, sondern mit Verweis auffehlendes Interesse am Thema Schmerzmanagement die Teilnahme aktiv ablehnten.

Aufgrund der hohen Spezialisierung und damit verbundenen Heterogenität der universitären Abteilungen erfolgte die Befragung dort in einem zweiten 
Tab. 5 Behandlungsstandards und Verantwortlichkeiten (universitäre Abteilungen)

Vorliegen von Behandlungsstandards ${ }^{a}$

\begin{tabular}{|c|c|}
\hline Schriftliche Standards & $54 \%(n=14)$ \\
\hline Mündliche Standards & $27 \%(n=7)$ \\
\hline Keine Standards & $19 \%(n=5)$ \\
\hline \multicolumn{2}{|c|}{ Integration von Bedarfsmedikation in den Standard ${ }^{b}$} \\
\hline $\mathrm{Ja}$ & $100 \%(n=14)$ \\
\hline \multicolumn{2}{|c|}{ Interventionstrigger für die Gabe einer Bedarfsmedikation ${ }^{c}$} \\
\hline $\mathrm{Ja}$ & $87 \%(n=13)$ \\
\hline Nein & $13 \%(n=2)$ \\
\hline \multicolumn{2}{|c|}{ Integration von nichtmedikamentösen Therapien in den Standard ${ }^{b}$} \\
\hline $\mathrm{Ja}$ & $36 \%(n=5)$ \\
\hline Nein & $64 \%(n=9)$ \\
\hline \multicolumn{2}{|c|}{ Verantwortlichkeit für die Überarbeitung der Standards ${ }^{b}$} \\
\hline Eigene Abteilung & $36 \%(n=5)$ \\
\hline Interdisziplinäre Arbeitsgruppe & $57 \%(n=8)$ \\
\hline Klinikweiter Schmerzbeauftragter & $7 \%(n=1)$ \\
\hline \multicolumn{2}{|c|}{ Effektivitätskontrolle der Schmerztherapie (Mehrfachantworten möglich) } \\
\hline Schmerzstärke in Ruhe & $70 \%(n=23)$ \\
\hline Analgetikaverbrauch & $52 \%(n=17)$ \\
\hline Nebenwirkungen & $52 \%(n=17)$ \\
\hline Vigilanz & $52 \%(n=17)$ \\
\hline $\begin{array}{l}{ }^{\mathrm{a}} \text { Fehlend: } \boldsymbol{n}=7 \\
{ }^{\mathrm{b}} \text { Fehlend: } \boldsymbol{n}=19 \\
{ }^{\mathrm{c}} \text { Fehlend: } \boldsymbol{n}=18\end{array}$ & \\
\hline
\end{tabular}

Schritt an alle Abteilungen adressiert mittels Onlinefragebogen, um die Übersicht über die Versorgungslandschaft $\mathrm{zu}$ ergänzen. Während von den teilnehmenden nichtuniversitären Abteilungen alle Teilnehmenden auch die Befragung abschlossen, brachen $36 \%$ der Kolleginnen und Kollegen der universitären Abteilungen die Onlinebefragung nach initialem Anklicken des Links im Verlauf der Beantwortung ab, sodass der Rücklauf für den vollständigen Fragebogen mit $11 \%$ der kontaktierten Abteilungen auch im Vergleich zu anderen Befragungen $\mathrm{zu}$ diesem Thema sehr niedrig war [13, 15, 21, 29]. Aufgrund des höheren Rücklaufs und auch durch die Möglichkeit, Fragen im persönlichen Gespräch zu erläutern, muss für die Daten der nichtuniversitären Krankenhäuser von einer höheren Aussagekraft ausgegangen werden, sodass diese im Fokus der Darstellung standen. Wenn auch ein Vergleich zwischen nichtuniversitären und universitären Krankenhäusern nicht direkt beabsichtigt war, ist er aufgrund des unterschiedlichen Befragungsmodus zudem eingeschränkt.
Vor dem Hintergrund, dass Schmerzen oft das erste Symptom sind, das zur internistischen Untersuchung und/oder stationären Aufnahme führt, ist es schon verwunderlich, dass sowohl in den nichtuniversitären als auch in den universitären Kliniken nur wenig Interesse hinsichtlich des Themas Schmerzmanagement bestand. In dieser Befragung wurden leider nur die Fachabteilungen erfasst und nicht dezidiert gefragt, ob die $\mathrm{Ab}$ teilungen auch Tumorpatienten behandeln. Es kann aber davon ausgegangen werden, dass eine Mehrheit der teilnehmenden Abteilungen und Kliniken sich auch mit onkologischen Patienten befasst und dass die Schmerztherapie auch unter diesem Aspekt eine wichtige Rolle spielen müsste.

\section{Struktur- und Prozessqualität}

Alles in allem skizzierte sich bei den teilnehmenden Abteilungen eine gute Implementierung von Prozessen und Strukturen des Schmerzmanagements. Bei der Wahrnehmung und regelmäßigen Erfassung von Schmerz als eigenständigem
Parameter zeigt sich ein deutlich besseres Bild im Vergleich zu der im Jahr 2012 im Rahmen des „Akutschmerzzensus" durch die anästhesiologischen Kollegen für die nichtoperativen Bereiche gemachten Einschätzung [13]. Während dort $57 \%$ der nichtoperativen Abteilungen angebenen hatten, Schmerzen nie standardmäßig zu erfassen, war dies in der vorliegenden Befragung nur in wenigen Abteilungen der Fall. Dennoch erfolgte in den meisten Abteilungen auch keine regelmäßige, mehrmals tägliche Erfassung (z. B. einmal pro Schicht) [13].

Diese positive Tendenz zeigte sich auch für die meisten anderen erfragten Prozess- und Strukturparameter zur Dokumentation, zu Behandlungsstandards und $\mathrm{zu}$ speziellen Versorgungsstrukturen des Schmerzmanagements wie beispielsweise dem Vorhandensein von Akutschmerzdiensten. Die Ergebnisse der hier vorgestellten Studie zeigen in den teilnehmenden Abteilungen ein annähernd ähnliches Bild der Implementierung von Strukturen und Prozessen des Schmerzmanagements, wie zuletzt in vielen Abteilungen der operativen Medizin [13].

Im Hinblick auf den Vergleich organisatorischer Anforderungen von Mitarbeitern der operativen und nichtoperativen Medizin an das Schmerzmanagement gibt es Hinweise, dass sich die organisatorischen Anforderungen gar nicht grundlegend unterscheiden [14]. Analog zur perioperativen Medizin konnten Einzelstudien zu prozedurenspezifischen Schmerzen in der konservativen Medizin zeigen, dass eine große Anzahl von Patienten postinterventionell unter mittelschweren bis schweren Schmerzen litten und diese durch ein strukturiertes Schmerzmanagement reduziert werden konnten [2, 7]. Allerdings lässt sich vermuten, dass sich die spezifischen Anforderungen an schmerzmedizinische Interventionen und Therapie zwischen den Patientenkollektiven unterscheiden und im Fall der internistischen Patienten über die in der operativen Medizin häufig im Vordergrund stehenden invasiven Analgesieverfahren (z. B. Periduralkatheter) oder opioidbasierten Therapieansätze hinausgehen [5, 12]. Bisher fehlen hierzu jedoch systematische Analysen und dem- 
Tab. 6 Spezielle Versorgungstrukturen und interdisziplinäre Zusammenarbeit (universitäre Abteilungen)

Verantwortlich für das Akutschmerzmanagement ${ }^{a}$

Stationsarzt und ASD (welcher zus. spezielle Verfahren betreut, z.B.PDK)

Stationsarzt und ASD (ohne weitere spezielle Verfah- $13 \%(n=3)$

ren)

Stationsarzt

$12 \%(n=3)$

Unterstützung der Abteilung bei der Betreuung von Patienten mit Schmerzen

$\mathrm{ASD}^{\mathrm{a}}$

$100 \%(n=20)$

Schmerzmedizinischer Konsildienst ${ }^{b}$

$100 \%(n=23)$

Palliativmedizinischer Konsildienst ${ }^{c}$

$100 \%(n=23)$

Schriftliche Vereinbarung zur Zusammenarbeit mit der schmerzmedizinischen Abteilung/dem schmerzmedizinischen Bereich ${ }^{d}$

Ja

Nein

$18 \%(n=4)$

Beantwortende/r wusste nicht, ob Vereinbarungen

$41 \%(n=9)$

vorlagen

$41 \%(n=9)$

ASD Akutschmerzdienst, PDK Periduralkatheter

Faehlend: $n=8$

${ }^{b}$ Fehlend: $\boldsymbol{n}=13$

'Fehlend: $\boldsymbol{n}=9$

${ }^{\mathrm{d}}$ Fehlend: $\boldsymbol{n}=10$

entsprechend die Einbeziehung in Leitlinien. Perspektivisch ist hier die Einbeziehung nichtoperativer Aspekte, z. B. in entsprechende Leitlinien und Empfehlungen, wünschenswert. Auch wurden diese Aspekte in der Befragung nicht systematisch erhoben.

Neben dem zu vermutenden erheblichen Selektionsbias gibt es aber auch andere Gründe, die für die Notwendigkeit einer Verbesserung der Versorgungssituation sprechen könnten. So fokussierte das Projekt „Schmerzfreies Krankenhaus“, aus welchem die Zertifizierung Certkom „Qualifizierte Schmerztherapie“ entstanden ist, von Anbeginn nicht nur auf die operative Medizin, sondern bezog sich auf die nichtoperativen Bereiche. Auch hier zeigte sich, dass initial unter den am Projekt bzw. später der Zertifizierung teilnehmenden nichtoperativen Abteilungen eine im Verhältnis zur operativen Medizin schlechtere Ergebnisqualität vorlag. Es konnte jedoch über die Jahre in den neu zu zertifizierenden Kliniken bzw. in der Rezertifizierung tendenziell eine Verbesserung beobachtet werden ([23, 24], Folgedaten nicht veröffentlicht). Zum anderen hat inzwischen die Implementierung des „Expertenstandards Schmerzmanagement in der Pflege" Verbreitung gefunden, und damit die Einbeziehung von Strukturen und Prozessen z. B. bzgl. des Schmerzassessments in der gesamten Klinik [5]. Immerhin ca. ein Drittel der Teilnehmenden berichtet, dass dieser in ihrer Abteilung implementiert sei. Auch zeichnet sich über die Jahre - auch in der Wahrnehmung aus Gesprächen mit Kolleginnen und Kollegen und der Autoren die fortschreitende Entwicklung einer engeren innerklinischen Zusammenarbeit zwischen Schmerzdiensten und internistischen Abteilungen ab. Dieses Potenzial wurde auch in der im Jahr 2019 veröffentlichten Definition und Empfehlung der Deutschen Gesellschaft für Anästhesiologie und Intensivmedizin e.V. (Nürnberg) zu personellen und qualifikatorischen Anforderungen von Schmerzdiensten berücksichtigt $[5,11]$.

\section{Qualitätssicherung und „patient-reported outcomes"}

Mit den Worten „If you can't measure it, you can't manage it" betonte der österreichisch-US-amerikanische Ökonom Peter Ferdinand Drucker die Bedeutung der Definition von Erfolg und der Erfassung der Ergebnisqualität bei Managementprozessen und Qualitätsbeurteilung [8]. In unserer Befragung zeigten sich bei den Interviews große Unsicherheiten in Bezug auf die Qualitätssicherung und die Zertifizierung des Schmerzmanagements in den Abteilungen. Diese Fragen konnten im persönlichen Gespräch teils geklärt werden, zusätzlich konnte für die telefonisch befragten Abteilungen die tatsächliche Zahl der zertifizierten Abteilungen ermittelt werden. Etwa die Hälfte der befragten Abteilungen gab initial an, dass die Schmerztherapie im Haus zertifiziert sei (• Tab. 3). Zum Zeitpunkt der Befragung (persönliche Anfrage beim TÜV Rheinland und bei der Deutschen Schmerzgesellschaft e.V. - seit dem Jahr 2017 Träger von Certkom; Stand Oktober 2018) waren in Deutschland und Österreich insgesamt 62 Krankenhäuser durch den TÜV Rheinland und 42 Krankenhäuser durch Certkom zertifiziert. Das entspricht bei 1942 deutschen Krankenhäusern (23.04.2020, DeStatis-Abfrage [3]) einem Anteil von $5 \%$ aller deutschen Krankenhäuser. Insgesamt waren innerhalb dieser Zertifizierungen deutschlandweit lediglich 53 internistische Abteilungen enthalten, was die Angaben zur Zertifizierung des Schmerzmanagements fraglich macht, da es unwahrscheinlich ist, dass bei der Randomisierung ein derart hoher Anteil der zertifizierten Kliniken eingeschlossen wurde (TÜV Rheinland $n=17$, Certkom e. V. $n=37$, hier angegeben insgesamt $n=22$ ). Auch der hohe Anteil universitärer Kliniken, die laut den Befragten ein zertifiziertes Schmerzmanagement hatten, ist fraglich bzw. lässt Zweifel an der Eindeutigkeit der Frage. Aufgrund der Anonymität der Umfrage konnten die Angaben für die nichtuniversitären Abteilungen nicht mit den Daten von Certkom e.V. und dem TÜV Rheinland abgeglichen werden. Vielmehr ist es wahrscheinlich, dass hier Unwissenheit vorlag oder diese sehr speziellen Zertifizierungen mit den Zertifizierungen von Organzentren, in denen auch oft Aspekte des Schmerzmanagements erfasst werden, verwechselt wurden.

Um den Qualitätsverbesserungsprozess des Schmerzmanagements zielgerichteter fortzusetzen, wäre eine regelmäßige Erfassung von „patient-reported outcomes“ (PRO), wie sie inzwischen 
in ca. 200 deutschen Krankenhäusern mit QUIPS (Qualitätsverbesserung in der postoperativen Schmerztherapie) erfolgt, auch in der internistischen Medizin sinnvoll. Hier besteht erhebliches Verbesserungs- bzw. Umsetzungspotenzial. Die Schwierigkeit der Befragung signalisiert aber auch, dass es wichtig ist, im breiten Kanon der internistischen Fächer die Abteilungen zu identifizieren, in denen ein solcher Aufwand sinnvoll und gewünscht ist. Unter den Teilnehmern erfolgt die Betrachtung von erhobenen Outcome-Parametern zur Qualitätssicherung des Schmerzmanagements nur in einem geringen Teil der Abteilungen. Das führt vermutlich $\mathrm{zu}$ „blinden Flecken“ und einer Unterschätzung der Relevanz, wie andere Veröffentlichungen vermuten lassen [6, 9, 14, 18-20, 24, 28, 31]. Auch in der operativen Medizin zeigte sich erst mit systemischen Erhebungen die Relevanz von Defizienten in der Ergebnisqualität des Schmerzmanagements [1, 16, 17]. Seit Kurzem existiert hierzu mit QUIKS (Qualitätsverbesserung im konservativen Schmerzmanagement) im Rahmen des QUIPS-Projekts auch ein Instrument zur systematischen Erfassung der Ergebnisqualität der Schmerzbehandlung in nichtoperativen Fachbereichen [9].

\section{Schlussfolgerung}

Insgesamt zeigten die internistischen Adressaten häufig ausdrückliches Desinteresse am Thema Schmerzmanagement. Unter den internistischen Abteilungen, die sich zur Teilnahme bereiterklärten, zeichneten sich jedoch gute Strukturen und Prozesse des Schmerzmanagements ab. Die Repräsentativität dieses Ergebnisses ist jedoch aufgrund geringer Teilnahme und hoher Ablehnung des Themas Schmerzmanagement kritisch zu bewerten und vermutlich von einem erheblichen Positivbias geprägt. Nichtuniversitäre und universitäre Kliniken scheinen sich nicht grundlegend $\mathrm{zu}$ unterscheiden, wenn auch aufgrund des deutlich niedrigeren Anteils vollständig erfasster universitärer Abteilungen diese Einschätzung nur vage getroffen werden kann. Bzgl. organisatorischer Rahmenbedingungen (z.B. Implementierung von Behandlungsstandards, schriftliche Vereinbarungen zur Zusammenarbeit, regelmäßige Qualitätssicherung) besteht selbst bei teilnehmenden Kliniken die Möglichkeit eines Verbesserungspotenzials. Insbesondere der Erfassung von PRO sollte zur regelmäßigen Evaluation und Verbesserung der Qualität des Schmerzmanagements in der internistischen Medizin eine größere Bedeutung zukommen.

\section{Fazit für die Praxis}

- In den Abteilungen für innere Medizin an universitären und nichtuniversitären Kliniken in Deutschland sollte dem Thema "Schmerzmanagement" mehr Interesse entgegengebracht werden.

- In den teilnehmenden Kliniken zeichneten sich gute Strukturen und Prozesse ab.

- Die Repräsentativität dieses Ergebnisses ist jedoch aufgrund geringer Teilnahme und hoher Ablehnung des Themas Schmerzmanagement kritisch zu bewerten und vermutlich von einem erheblichen Positivbias geprägt.

- Verbesserungspotenzial zeigte sich in den teilnehmenden Kliniken vor allem bei den organisatorischen Rahmenbedingungen wie der Implementierung von Behandlungsstandards, schriftlichen Vereinbarungen und regelmäßiger Qualitätssicherung.

- Insbesondere der Erfassung von „patient-related outcomes“ (PRO) sollte zur regelmäßigen Evaluation und Verbesserung der Qualität des Schmerzmanagements in der internistischen Medizin eine größere Bedeutung zukommen.

\section{Korrespondenzadresse}

\section{Dr. med. M. I. Emons}

Klinik für Anästhesiologie, Universitätsmedizin Göttingen

Robert-Koch-Str. 40, 37075 Göttingen,

Deutschland

miriam.emons@med.uni-goettingen.de

Förderung. Die Finanzierung dieser Studie erfolgte aus Personal- und Sachmitteln der Klinik für Anästhesiologie der Universitätsmedizin Göttingen.
Funding. Open Access funding enabled and organized by Projekt DEAL.

\section{Einhaltung ethischer Richtlinien}

Interessenkonflikt. M.I. Emons, T.H. Scheeper-von der Born, V. Ellenrieder und L. Reinhardt geben an, dass kein Interessenkonflikt besteht. F. Petzke ist am Projekt QUIKS - Qualitätsverbesserung im konservativen Schmerzmanagement, dem Modul für nichtoperative Fachbereiche des QUIPS-Projekts, wissenschaftlich beteiligt. W. Meißner gibt an, unabhängig von der eingereichten Arbeit folgende Zuwendungen erhalten zu haben: Forschungsgelder und persönliche Honorare von Grünenthal, Forschungsgelder von Pfizer, persönliche Honorare von TAD, persönliche Honorare von BioQ Pharm, persönliche Honorare von Bionorica, persönliche Honorare von Kyowa, persönliche Honorare von Northern Swan, Forschungsgelder von Mundipharma, persönliche Honorare von Tilray. Er ist am Projekt QUIKS - Qualitätsverbesserung im konservativen Schmerzmanagement, dem Modul für nichtoperative Fachbereiche des QUIPS-Projekts, wissenschaftlich beteiligt. Darüber hinaus ist W. Meißner als Projektleiter von QUIPS beteiligt. J. Erlenwein ist 2. Sprecher des Arbeitskreises Akutschmerz und Mitglied der Ad-hocKommission Zertifizierung der Deutschen Schmerzgesellschaft e. V., Berlin. Außerdem ist er 1. Sprecher des Wissenschaftlichen Arbeitskreises Schmerzmedizin der Deutschen Gesellschaft für Anästhesiologie und Intensivmedizin e. V., Nürnberg. Er ist am Projekt QUIKS - Qualitätsverbesserung im konservativen Schmerzmanagement, dem Modul für nichtoperative Fachbereiche des QUIPS-Projekts, wissenschaftlich beteiligt.

Ein Ethikvotum war für die vorliegende Arbeit nicht nötig. Es wurden in der vorliegenden Arbeit keine Studien an Menschen oder Tieren durchgeführt. Es handelt sich um eine anonymisierte Befragung ärztlicher bzw. pflegerischer Kollegen zur Praxis der Schmerztherapie in der jeweiligen Abteilung. Es wurde nicht auf Patientendaten zurückgegriffen, sondern lediglich auf die Aussage der Kollegen zur gängigen Praxis der Schmerzerfassung und Therapie.

Open Access. Dieser Artikel wird unter der Creative Commons Namensnennung 4.0 International Lizenz veröffentlicht, welche die Nutzung, Vervielfältigung, Bearbeitung, Verbreitung und Wiedergabe in jeglichem Medium und Format erlaubt, sofern Sie den/die ursprünglichen Autor(en) und die Quelle ordnungsgemäß nennen, einen Link zur Creative Commons Lizenz beifügen und angeben, ob Änderungen vorgenommen wurden.

Die in diesem Artikel enthaltenen Bilder und sonstiges Drittmaterial unterliegen ebenfalls der genannten Creative Commons Lizenz, sofern sich aus der Abbildungslegende nichts anderes ergibt. Sofern das betreffende Material nicht unter der genannten Creative Commons Lizenz steht und die betreffende Handlung nicht nach gesetzlichen Vorschriften erlaubt ist, ist für die oben aufgeführten Weiterverwendungen des Materials die Einwilligung des jeweiligen Rechteinhabers einzuholen.

Weitere Details zur Lizenz entnehmen Sie bitte der Lizenzinformation auf http://creativecommons.org/ licenses/by/4.0/deed.de. 


\section{Literatur}

1. Apfelbaum JL, Chen C, Mehta SS et al (2003) Postoperative pain experience: results from a national survey suggest postoperative pain continues to be undermanaged. Anesth Analg 97:534-540 (table of contents)

2. Bode K, Breithardt OA, Kreuzhuber M et al (2015) Patient discomfort following catheter ablation and rhythm device surgery. Europace 17:1129-1135

3. Destatis (2020) Grunddaten der Krankenhäuser. Statistisches Bundesamt. https://www. destatis.de/DE/Themen/Gesellschaft-Umwelt/ Gesundheit/Krankenhaeuser/_inhalt.html.Zugegriffen:23.04.2020

4. Deutsche Krankenhaus TrustCenter und Informationsverarbeitung Dktig Deutsches Krankenhausverzeichnis. https://www.deutscheskrankenhaus-verzeichnis.de/. Zugegriffen: 05.07.2017

5. Dnqp (2011) Expertenstandard Schmerzmanagement in der Pflege bei akuten Schmerzen. Deutsches Netzwerk für Qualität in der Pflege, Osnabrück

6. Donovan M, Dillon P, Mcguire L (1987) Incidence and characteristics of pain in a sample of medicalsurgical inpatients. Pain 30:69-78

7. Dorschner M, Bollmann A, Dinov B et al (2017) Structured pain management reduces patient discomfort after catheter ablation and rhythm device surgery. Eur J Pain 21:1723-1731

8. Drucker PF (2000) Die Kunst des Managements: eine Sammlung der in der Harvard Business Review erschienenen Artikel. Econ, Düsseldorf

9. Erlenwein J, Bertemes C, Kunsch S et al (2020) Qualitatsverbesserungimkonservativen Schmerzmanagement (QUIKS): Ein Modul des QUIPSProjekts zum Benchmarking der Schmerztherapie bei Patienten der nichtoperativen Versorgung. Schmerz 34:52-64

10. Erlenwein J, Koschwitz R, Pauli-Magnus D et al (2016) A follow-up on acute pain services in Germany compared to international survey data. Eur J Pain 20:874-883

11. Erlenwein J, Meissner W, Petzke F et al (2019) Personelle und organisatorische Voraussetzungen fur Schmerzdienste in Krankenhausern: Empfehlung der Deutschen Gesellschaft fur Anasthesiologie und Intensivmedizin e.V. Anästh Intensivmed 60:265-272

12. Erlenwein J, Schlink J, Pfingsten $M$ et al (2012) Klinische Schmerzkonsile: Profile klinischer Schmerzkonsile und Anforderungen komplexer Schmerzpatienten an die innerklinischen Versorgungsstrukturen. Schmerz 6:692-698

13. Erlenwein J, Stamer U, Koschwitz R et al (2014) Akutschmerztherapie in der stationären Versorgung an deutschen Krankenhäusern-Ergebnisse des Akutschmerzzensus 2012. Schmerz 28:147-156

14. Erlenwein J, Ufer G, Hecke A et al (2013) Anforderungen an die Organisation der Schmerztherapie im Krankenhaus: Abteilungsubergreifender Vergleich von Anforderungen an das Schmerzmanagement aus Mitarbeitersicht. Schmerz 27:553-565

15. Erlenwein J, Waeschle RM, Bauer $M$ et al (2016) Finanzierung von Akutschmerzdiensten an deutschen Krankenhäusern. Anästh Intensivmed 57:246-256

16. Gan TJ, Habib AS, Miller TE et al (2014) Incidence, patient satisfaction, and perceptions of postsurgical pain: results from a US national survey. Curr Med Res Opin 30:149-160
17. Gerbershagen $\mathrm{HJ}$, Aduckathil $\mathrm{S}$, van Wijck $A J$ et al (2013) Pain intensity on the first day after surgery: a prospective cohort study comparing 179 surgical procedures. Anesthesiology 118:934-944

18. Gu X, Belgrade MJ (1993) Pain in hospitalized patients with medical illnesses. J Pain Symptom Manage 8:17-21

19. Helfand M, Freeman M (2009) Assessment and management of acute pain in adult medical inpatients: a systematic review. Pain Med 10:1183-1199

20. Korczak D, Kuczera C, Rust M (2013) Akutschmerztherapie auf operativen und konservativen Stationen. Deutsches Institut für Medizinische Dokumentation und Information, Köln

21. Lassen $\mathrm{CL}$, Link $\mathrm{F}$, Lindenberg $\mathrm{N}$ et al (2013) Anästhesiologische Akutschmerztherapie in Deutschland:Telefonbasierte Umfrage. Anaesthesist 62:355-364

22. Laubenthal H (2007) Leitlinie „Behandlung akuter perioperativer und posttraumatischer Schmerzen". Arbeitsgemeinschaft der Wissenschaftlich Medizinischen Fachgesellschaften, Frankfurt a.M.

23. Maier C, Nestler N, Hansel $N$ et al (2013) Zertifizierung für "Qualifizierte Schmerztherapie" - Nachhaltige Verbesserung der Versorgungsrealität in deutschen Krankenhäusern. Klinikarzt 42:80-87

24. MaierC, Nestler N, Richter Het al (2010) Qualität der Schmerztherapie in deutschen Krankenhäusern. Dtsch Arztebl Int 107:607-614

25. MeissnerW, Komann M, Erlenwein Jet al (2017) The quality of postoperative pain therapy in German hospitals. Dtsch Arztebl Int 114:161-167

26. Neugebauer E (2007) Editorial: Akutschmerztherapie im Aufwind. Schmerz 21:501-502

27. Neugebauer E, Hempel K, Sauerland S et al (1998) Situation der perioperativen Schmerztherapie in Deutschland. Ergebnisse einer repräsentativen, anonymen Umfrage von 1000 chirurgischen Kliniken. AG Schmerz. Chirurg 69:461-466

28. Salomon L, Tcherny-Lessenot S, Collin Eet al (2002) Pain prevalence in a French teaching hospital. JPain Symptom Manage 24:586-592

29. Stamer U, Mpasios N, Stuber F et al (2002) Postoperative Schmerztherapie in Deutschland. Ergebnisse einer Umfrage. Anaesthesist 51:248-257

30. Stamer UM, Mpasios N, Stuber F et al (2002) A survey of acute pain services in Germany and a discussion of international survey data. Reg Anesth Pain Med 27:125-131

31. Strohbuecker B, Mayer H, Evers GC et al (2005) Pain prevalence in hospitalized patients in a German university teaching hospital. J Pain Symptom Manage 29:498-506

\section{SEdeditorial
Manager}

\section{Hilfestellungen für den Editorial Manager}

Das Einreichungs- und Begutachtungssystem Ihrer Zeitschrift

Sowohl für die ganz alltäglichen Fragen in der Handhabung des Editorial Managers als auch für spezielle Problematiken finden Sie auf www.springermedizin.de/editorialmanager eine Vielzahl an Handreichungen, die Ihnen die Arbeit als Gutachter*in, Autor*in oder Herausgeber*in erleichtern.

Über Videos, einseitige Schritt-für-SchrittAnleitungen oder ein umfangreiches Manual werden Sie durch die einzelnen Punkte geführt, wie:

- Wie reiche ich ein Manuskript ein?

- Wie finde ich passende Gutachter*innen?

- Wie lade ich Gutachter*innen ein?

- Wie nehme ich ein Gutachten an bzw. lehne es ab?

- Wo erkenne ich, in welchem Status ein Mansukript ist?

- Wie ändere ich meine persönlichen Informationen?

- Wo kann ich meinen Urlaub eintragen? 